\title{
Computed Tomography Radiomics for the Preoperative Prediction of Cervical Lymph Node Metastasis in Papillary Thyroid Carcinoma: Development and External Validation
}

\section{Zhi-Jiang Han ( $\sim$ hzj1022@zju.edu.cn )}

Department of Radiology, Affiliated Hangzhou First People's Hospital, Zhejiang University School of Medicine, Hangzhou, China

\section{Peiying Wei}

Department of Radiology, Affiliated Hangzhou First People's Hospital, Zhejiang University School of Medicine, Hangzhou, China

\section{Zhongxiang Ding}

Department of Radiology, Key Laboratory of Clinical Cancer Pharmacology and Toxicology Research of Zhejiang Province, Affiliated Hangzhou First People's Hospital, Zhejiang University School of Medicine, Hangzhou, China

\section{Dingcun Luo}

Department of Surgical Oncology, Affiliated Hangzhou First People's Hospital, Zhejiang University School of Medicine, Hangzhou, China

\section{Liping Qian}

Department of Radiology, Hangzhou Cancer Hospital, Hangzhou, China

\section{Jian Zhou}

Department of Radiology, Hangzhou Cancer Hospital, Hangzhou, China

\section{Haibin Wang}

Department of Radiology, Affiliated Hangzhou First People's Hospital, Zhejiang University School of Medicine, Hangzhou, China

\section{Wenli Cai}

Department of Radiology, Massachusetts General Hospital, Massachusetts, United States

\section{Ming Zhang}

Department of Medical Imaging, The First Affiliated Hospital of Xi'an Jiaotong University, Xi'an, China

\section{Research article}

Keywords: Papillary thyroid carcinoma, lymph node metastasis, computed tomography, radiomics 
DOI: https://doi.org/10.21203/rs.3.rs-26914/v1

License: (c) (i) This work is licensed under a Creative Commons Attribution 4.0 International License. Read Full License 


\section{Abstract}

Background Cervical lymph node (LN) status is a critical factor related to the treatment and prognosis of papillary thyroid carcinoma (PTC). The aim of this study was to investigate the preoperative predictions of cervical LN metastasis in PTC using computed tomography (CT) radiomics.

Methods A total of 134 PTC patients who underwent CT examinations were enrolled in the study at two institutes between January 2018 and January 2020. Of these patients, 289 cervical LNs (institute 1: 206 LNs from 88 patients; institute 2: 83 LNs from 46 patents) were selected. All the cases had been confirmed by surgery and pathology. Each LN was segmented and 1408 radiomic features were calculated radiomic features in noncontrast and contrast-enhanced CT images. Features were selected using the Boruta algorithm followed by an iterative culling-out algorithm. We compared four machine learning classifiers, including random forest (RF), support vector machine (SVM), neural network (NN), and naïve bayes (NB) for the classification of LN metastasis. The models were first trained and validated by 10 -fold cross-validation using data from institute 1 and then tested using independent data from institute 2. The performance of the models was compared using the area under the receiver operating characteristic curves (AUC).

Results Seven radiomic features were selected for building the models - 3 histogram statistical textures, 1 gray level co-occurrence matrix texture, and 3 gray level zone size matrix textures. The AUCs of the radiomic models with 10-fold cross-validation were 0.941 (95\% confidence interval [Cl]: 0.93-0.95), 0.943 (95\% Cl: 0.93-0.95), 0.914 (95\% Cl: 0.90-0.95), and 0.905 (95\% Cl: 0.88-0.91) for RF, SVM, NN, and NB, respectively. The AUCs for the testing data were 0.926 (95\% Cl: 0.86-0.98), 0.932 (95\% Cl: 0.88-0.98), 0.925 (95\% Cl: $0.86-0.97)$, and 0.912 (95\% Cl: 0.83-0.98) for RF, SVM, NN, and NB, respectively.

Conclusions $\mathrm{CT}$ radiomic model demonstrated robustness in preoperative classification of LN metastases for patients with PTC, which may provide significant support for clinical decision making and prognosis evaluation.

\section{Background}

Thyroid carcinoma is the most common malignant tumor of endocrine system and one of the fastestgrowing tumors in the world[1]. Among the thyroid carcinoma, papillary thyroid carcinoma (PTC) accounts for approximately $90 \%[2,3]$. Although the 5- and 10-year survival rates of low-risk PTC patients approach $100 \%, 30-90 \%$ of them are associated with cervical lymph node (LN) metastasis at the time of diagnosis $[4,5]$. LN metastasis is the most important risk factor for local recurrence, which is more severe than the primary lesion [6]. Resection may be performed twice or more due to local recurrence, which affects the life quality of patients. Therefore, accurate preoperative identification of cervical LN metastasis is crucial to the selection of surgical methods and the prediction of local tumor recurrence, especially in high-risk groups with PTC metastasis [7, 8]. 
Ultrasound (US) is a common imaging modality in the preoperative assessment of cervical LN metastasis in PTC. It is limited by operator dependence and examination field. In addition, US is affected by the concealment of the trachea, esophageal gas, and sternum $[9,10]$. Previous studies have shown that the specificity and sensitivity of US for the diagnosis of PTC cervical LN metastasis are $85.0 \%-97.4 \%$ and $36.7 \%-61.0 \%$, respectively [11-13]. CT overcomes the deficiencies of US and permits more comprehensive and intuitive visualizations of lesions and their relationships with surrounding structures. In addition, the combination of noncontrast and contrast-enhanced CT allows better visualization and evaluation of the internal microcirculation of LNs [14]. Current guidelines recommend CT examination when US assessment is inadequate, large, or extensive for $L N$ metastasis $[15,16]$. However, the sensitivity of traditional CT diagnosis method for predicting PTC cervical LN metastasis is also insufficient, approximately $55 \%-62 \%[17,18]$ In addition, the interpretation of image signs is highly subjective and diagnoses may not be accurate.

Radiomics may be used with large amounts of image data for thorough extraction and analysis of relevant features from regions of interest (ROI) in images. Machine learning and statistical analyses can be used to extract key information and establish objective analysis models. This can improve clinical diagnosis, which is currently highly subjective, consequently, and less evidence-based. The prediction model can assist clinical diagnosis and prognostic analysis and facilitate more accurate diagnoses and effective treatments $[19,20]$. The predictive value of PTC primary lesion on cervical LN metastasis by CT radiomic model has been reported [21]. However, to the best of our knowledge, there is rare reliable report of CT radiomics to predict cervical LN metastasis in PTC by analyzing LN itself. Based on our previous studies [22-24], this study established a CT radiomic model for predicting cervical LN metastasis from PTC. Internal and external cross-validation were conducted for the first time to explore the reliability of CT radiomic models in predicting cervical $L N$ metastasis

\section{Methods}

\section{Study subjects}

We retrospectively reviewed data of PTC patients at two institutes between January 2018 and January 2020. The inclusion criteria were: (i) An adult patient over 18 years old, who has not received surgery and antitumor treatment, (ii) PTC was confirmed by surgical pathology and LN surgical pathology was obtained by cervical LN dissection, (iii) preoperative noncontrast and contrast-enhanced CT scans were performed within 2 weeks prior to surgery and biopsy. The exclusion criteria were as follows: (i) incomplete clinical and imaging data, (ii) unclear LN display due to image quality or other problems.

In terms of our previous study and other reports [25-28], the following inclusion criteria were established for metastatic LNs: (i) no other definite cervical LN lesion, such as tuberculosis and lymphoma; (ii) selected from the cervical LN group with two LN metastases confirmed by pathology; (iii) short diameter of $L N \geq 5 \mathrm{~mm}$; (iiii) the $L N$ with highest score and $\geq$ two points (the classic signs of PTC LN metastasis including maximum short diameter, short diameter/long diameter $\geq 1 / 2$, highest enhancement, cystic 
degeneration/necrosis, and microcalcification were assigned one point, respectively). The inclusion criteria for non-metastatic LNs met the following three points: (i) no other definite cervical LN lesion, such as tuberculosis and lymphoma; (ii) selected from the cervical LN groups with no LN metastasis confirmed by pathology; (iii) the $\mathrm{LN}$ with largest diameter and a short diameter $\geq 5 \mathrm{~mm}$ within the groups mentioned in point (ii).

\section{Ct Examinations}

Each patient underwent noncontrast and contrast-enhanced CT examinations in the supine position with a 16-layer CT scanner (Institute 1: Lightspeed, GE, United States; Institute 2: Siemens Healthineers, German). The scanning range was from oropharynx to superior clavicle. The contrast agent (Institute 1: Bayer, German; Institute 2: Yangtze River, China) was injected intravenously through the elbow with a high-pressure syringe. The specific parameters of the two institutes were listed in the Table 1.

Table 1

CT scan parameters of the two institutes

\begin{tabular}{|lll|}
\hline Parameters & Institute 1 & Institute 2 \\
\hline Tube voltage $(\mathrm{kV})$ & 120 & 120 \\
\hline Tube current $(\mathrm{mA})$ & 250 & 250 \\
\hline Collimation $(\mathrm{mm})$ & $0.625 \times 16$ & $0.625 \times 16$ \\
\hline Pitch & 0.938 & 1.1 \\
\hline Rack rotation time $(\mathrm{s})$ & 0.5 & 0.5 \\
\hline Slice thickness $(\mathrm{mm})$ & 3.75 & 3.0 \\
\hline Contrast agent dose $(\mathrm{mL})$ & $50-60$ & $70-80$ \\
\hline lodine concentration $(\mathrm{mgl} / \mathrm{mL})$ & 370 & 300 \\
\hline Injection rate $(\mathrm{mL} / \mathrm{s})$ & $1.5-2.0$ & $2.5-3.0$ \\
\hline Contrast-enhanced CT time (s) & 50 & 50 \\
\hline $\begin{array}{l}\text { Note: Contrast-enhanced CT time: Contrast-enhanced CT } \\
\text { injection. }\end{array}$ & & \\
\hline
\end{tabular}

\section{Ln Segmentation}

The noncontrast and contrast-enhanced CT images of patients were retrieved from the picture archiving and communication system and imported to an open volumetric image analysis platform 3DQI (developed by the 3D quantitative imaging laboratory at Massachusetts General Hospital and Harvard Medical School). It facilitated data loading, segmentation, feature calculation, feature selection and 
building of the radiomic model. One radiologist (P.W. with 5 years of experience) manually contoured the target LN in the contrast-enhanced CT image, slice by slice, and used them as the standard to delineate the LN at the same level on the noncontrast CT scan to maintain consistency with the contrast-enhanced image, while exercising caution to avoid the surrounding blood vessels, calcification, peripheral fat, and other non-LN tissues. The corresponding sagittal and coronal planes of the LN were referenced when it was ambiguous in the axial plane. The segmentation results were checked by a senior head and neck radiologist (Z.H. with 18 years of experience in head and neck radiology). Any disagreement between the two radiologists was resolved by discussion and consensus. Both radiologists were blinded to the pathological assessment of $L N$ after surgery.

\section{Radiomic Features Extraction And Analysis}

Radiomic feature extraction and analysis were carried out on the 3DQI platform. Five categories of volumetric textures were calculated for the segmented $L N$ including 11 shape features, 25 histogram statistical textures, 22 gray level co-occurrence matrix (GLCM) textures, 16 gray-level run-length matrix (GLRLM) textures, and 14 gray level zone size matrix (GLZSM) textures. A 3D, discrete, and single-stage wavelet transform was used to decompose volumetric images into eight decomposed volumes of images, labeled as LLL, LLH, LHL, LHH, HLL, HLH, HHL, and HHH, where $L$ and $H$ are low- and highfrequency signals, respectively. In the eight decomposed volumes of images, 3DQI calculated four categories of volumetric textures except for the shape features in the segmented LN. This resulted in a total of 704 texture features for each segmented LN, including 88 volumetric textures plus $616(8 \times 77)$ wavelet-transformed textures in one scan. CT images of each LN included two phases of pre-contrast and post-contrast, which were used to calculate the texture features of the two phases respectively; a total of 1408 texture features.

Important features that would be useful for classification and image recognition were selected from a large number of texture features for modeling. We adopted a two-round feature selection method to select important features for the classification of benign and malignant LNs. First, the importance scores calculated by the Boruta algorithm were used for a rapid reduction of texture dimensionality [29]. The Boruta algorithm is a feature ranking and selection algorithm based on the random forest algorithm, which identifies all features which are either strongly or weakly related to the decision variable. Nonrelevant features were rejected using a Z-score cutoff of less than 0.01 . During the second round, an iterative culling-out algorithm was used to refine the performance of a classifier; an RF model was used [30]. In each iteration, we calculated the classification performance of the model by removing one of the textures, which characterize the area under the receiver operating characteristic (ROC) curves (AUC). If an AUC value, using one less texture parameter, was higher than that of the current model, the model with the maximum AUC value was selected. This iteration continued until the current model had the highest AUC.

We compared four machine-learning classification algorithms, including random forest (RF), support vector machine (SVM), neural network (NN), and naïve bayes (NB) and determined the optimal algorithm 
for the radiomic model. The performance of the model was measured by AUC. We used the 10 -fold crossvalidation to validate the model during training. For the 10 -fold cross-validation, the entire dataset was randomly divided into 10 subsets. Nine subsets were used for training the classification model. Subsequently, the trained models were tested with the subset that was not used for training. This procedure was repeated for each subset until all the subsets had been tested. This 10 -fold crossvalidation was repeated 10 times to optimize and stabilize the performance of the models. In addition, the built models were tested with the external dataset from institute 2. We compared the performances of the models using AUC, sensitivity, specificity, and accuracy on the classification of benign and malignant LNs.

\section{Statistical analysis}

The statistical methods used in feature selection and model construction were provided by 3DQI software. SPSS 22.0 was used to analyze general data. The Kolmogorov-Smirnov test was used to test the normality of age and mean \pm standard deviation (SD) were used for the normal distribution. In addition, the t-test was performed. The median (interquartile range) was used for non-normal distribution, and the Mann-Whitney test was adopted. Pearson's chi-square test or Fisher's exact test was used to compare the gender differences between the two groups. $P<0.05$ was statistically significant.

\section{Results}

\section{Baseline Characteristics}

Of 206 LNs from 88 PTC patients at institute 1, we identified 103 benign and 103 malignant LNs, respectively. Of 83 LNs from 46 PTC patients at institute 2, there were 41 benign and 42 malignant LNs. The data from institute 1 were used for model training, and the data from institute 2 were used for external validation.

In institute 1, there were 33 patients in the metastatic group with a total of $103 \mathrm{LNs}$, including 11 males and 22 females, aged 17-71 years, with an average age of 39 (35-60) years. In the non-metastatic group, there were 55 patients with a total of $102 \mathrm{LNs}$, including 22 males and 33 females, aged 24-70 years, with an average age of $44(36-53)$ years. There was no significant difference in gender $(P=0.532)$ and age $(P=0.766)$ between the two groups.

In institute 2, there were 19 patients in the metastatic group with a total of $41 \mathrm{LNs}$, including 6 males and 13 females, with an age range of 21 to 79 years and average of $45.26 \pm 16.28$ years. In the nonmetastatic group, there were 31 patients with a total of $42 \mathrm{LNs}$, including 5 males and 26 females, with an average age of $49.81 \pm 12.22$ years and ranging from 25 to 77 years. There was no significant difference in gender $(P=0.201)$ and age $(P=0.267)$ between the two groups.

\section{Performance Of Radiomic Models}


Seven important features were selected from 1408 pre-contrast and contrast-enhanced CT images (Fig. 1) -3 histogram features, 1 GLCM feature, and 3 GLZSM features. The three histogram features were 0_HIST_quant0.75 (pre-contarst CT histogram, 75\% quantile of the voxel intensity), 1_HIST_quant0.975 (contrast-enhanced CT histogram, 97.5\% quantile of the voxel intensity), and 1_HIST_quant0.75 (contrastenhanced CT histogram, quantile of the voxel intensity). The GLCM feature was 0_GLCM_clusShade (Cluster Shade of noncontrast CT, which is a measure of the skewness and uniformity of the GLCM). The three GLZSM features were 0_GLZSM_sae (Small Area Emphasis of noncontrast CT: a measure of the distribution of small size zones), 1_GLZSM_gln (Gray Level Non-Uniformity of contrast-enhanced CT: a measure of the variability of gray-level intensity values in the image), and 1_GLZSM_ze (Zone Entropy of contrast-enhanced CT: a measure of the uncertainty/randomness in the distribution of zone size and gray levels). Five texture features (0_HIST_quant0.75, 1_HIST_quant0.975, 1_HIST_quant0.75, 0_GLCM_ clusShade, and 1_GLZSM_gln) of LNs in the metastatic group were higher than those in the nonmetastatic group. In contrast, two texture features (1_GLZSM_ze and 0_GLZSM_sze) of LNs in the nonmetastatic group were higher than those in the metastatic group.

Figure 2A shows the ROCs of four radiomic models using RF, SVM, NN, and NB. The AUCs of these four radiomic models with 10-fold cross-validation were 0.941 (95\% confidence interval [Cl]: 0.93-0.95), 0.943 (95\% Cl: 0.93-0.95), 0.914 (95\% Cl: 0.90-0.95), and 0.905 (95\% Cl: 0.88-0.91) for RF, SVM, NN, and NB, respectively. The sensitivity, specificity, and accuracy of these models are listed in Table 2.

Table 2

Performance of the radiomic algorithms for metastatic lymph nodes in 10fold cross-validation and external validation

\begin{tabular}{|lllll|}
\hline Algorithm & \multicolumn{4}{l}{ 10-fold cross-validation } \\
\cline { 2 - 5 } & AUC & Sensitivity & Specificity & Accuracy \\
\hline Random forest & 0.941 & 0.886 & 0.862 & 0.874 \\
\hline Support vector machine & 0.943 & 0.906 & 0.855 & 0.881 \\
\hline Neural network & 0.914 & 0.769 & 0.884 & 0.827 \\
\hline Naïve bayes & 0.905 & 0.951 & 0.691 & 0.821 \\
\hline Algorithm & External validation & & \\
\cline { 2 - 5 } & AUC & Sensitivity & Specificity & Accuracy \\
\hline Random forest & 0.926 & 0.780 & 0.905 & 0.843 \\
\hline Support vector machine & 0.932 & 0.951 & 0.786 & 0.867 \\
\hline Neural network & 0.925 & 0.854 & 0.786 & 0.819 \\
\hline Naïve bayes & 0.912 & 0.854 & 0.857 & 0.855 \\
\hline
\end{tabular}


Figure 2B shows the ROCs of the four radiomic models using RF, SVM, NN, and NB and data from institute 2. The external validation revealed AUC of 0.926 (95\% Cl: $0.86-0.98), 0.936$ (95\% Cl: $0.88-0.98)$, 0.925 (95\% Cl: 0.86-0.97), and 0.912 (95\% Cl: 0.83-0.98) for RF, SVM, NN, and NB, respectively. The sensitivity, specificity, and accuracy of these models are listed in Table 2.

\section{Discussion}

In this study, we used the 3DQI software to extract the features of PTC cervical LNs at two institutes and select the feature parameters closely related to $L N$ metastasis, establishing a CT radiomic model to predict $L N$ metastasis based on these parameters. An internal 10-fold cross-validation showed that the AUC and accuracy were slightly higher in RF and SVM than NN and NB; SVM had a higher sensitivity, while RF had a slightly higher specificity. External validation also showed similar results, indicating the stability of the model. The model had satisfactory performance in the preoperative prediction of PTC cervical LN metastasis.

Currently, the preoperative CT evaluation of PTC cervical LN metastasis mainly relies on the signs such as microcalcification, necrotic or cystic degeneration, uniform or non-uniform high enhancement, and minimum/maximum diameter $>0.5[25,26]$. However, subjective evaluation of these signs by radiologists has limited the performance for the diagnosis of LN metastasis. Previous studies reported that the sensitivity was approximately $55 \%-62 \%$, and the specificity was $87 \%[17,18]$, which are significantly lower than the results of our study. In recent years, radiomics have been applied in the evaluation of PTC cervical LN metastasis mainly based on US image analysis [5, 31, 32]. Kim et al. [31] and Liu et al. [32] used US texture analysis to evaluate the predictive value of PTC primary lesions for cervical LN metastasis and obtained the opposite conclusion. We speculate that this might be related to the high operator dependence of US, in addition to the inclusion criteria of the two samples.

There are fewer studies on CT-based radiomic assessment of cervical LN metastasis in PTC. Lu et al. [21] analyzed the performance of CT radiomics for predicting cervical LN metastasis from a PTC primary lesion and found that the clinical nomogram yielded an AUC of 0.867 when incorporating the radiomic signature. Lee et al. [33] predicted cervical LN metastasis in thyroid cancer using CT deep learning. The AUC of the best-performance algorithm was 0.953 , which was similar to our results. However, we employed machine-learning radiomic methods and first performed external validation for model reliability. In addition, multiple cervical LN metastasis were common in PTC patients, but CT could hardly reach the node-node level comparison between LN imaging and pathology. In this study, we first proposed to score the classic signs of PTC LN metastasis and classified the LN with highest score as metastatic $\mathrm{LN}$, thus maximizing close to the comparison of lymph node imaging and pathology. As for nonmetastatic LNs, we selected the largest one from pathologically confirmed non-metastatic LN group to ensure the reliability and repeatability of our study.

The radiomic features of $L N$ images extracted in the study included histogram features, GLCM and GLXSM texture features, in both pre-contrast and contrast-enhanced CT. The histogram features, 
0_HIST_quant0.75, 1_HIST_quant0.975, and 1_HIST_quant0.75, reflected intensity information in a given $\mathrm{ROI}$ and quantified the heterogeneity within the $\mathrm{LN}$, which can also represent lesion volume. Our results showed that the above histogram features of LNs in the metastatic group were higher than those in the non-metastatic group, indicating increased heterogeneity and volume in the metastatic LNs. The 0_GLCM_clusShade feature of GLCM can represent the uniformity and skewness of CT value spatial distributions within LNs that is mainly reflected in the influence of pixels with spatial dependence and their relationship with surrounding environments [34]. A higher cluster shade texture implies more asymmetry and heterogeneous pixels in metastatic than non-metastatic LNs. 0_GLZSM_sze,

1_GLZSM_ze, and 1_GLZSM_gln were GLZSM textures, which represented the small zone emphasis, zone entropy, and gray-level non-uniformity. A lower GLZSM_sze in pre-contrast and GLZSM_ze in contrastenhanced images indicate that non-metastatic LNs have more small zone or more fine textures, and more randomness in the contrasted-enhanced images, whereas a higher GLZSM_gln texture indicates metastatic LNs are more heterogeneous in texture patterns. The Boruta algorithm was used to screen the features. The goal of feature selection was to obtain the feature set that can minimize the loss function of the current model and the goal of the Boruta algorithm was to screen out all the feature sets that were relevant to the dependent variables so that the influence factors of the dependent variables could be considered more comprehensively. The model was trained and validated with 10 iterations of 10 -fold cross-validation to avoid model overfitting. Subsequently, model testing was carried out with external data to ascertain real-world performance.

Our study had two limitations. First, LNs with a short diameter $<5 \mathrm{~mm}$ were not included to avoid the partial volume effect of scans since the slice thickness of CT scan was 3-3.75 mm. Therefore, further studies are needed to evaluate model performance on these small nodules. Second, our study was retrospective at only two medical institutes. Further prospective studies with a larger sample size of patients from more institutions are required to validate our results.

\section{Conclusions}

The CT radiomic model showed high diagnostic performance for predicting PTC cervical LN metastasis and provided support to clinicians for accurate preoperative evaluation and treatment of LN metastasis.

\section{Abbreviations}

PTC: papillary thyroid carcinoma; LN: lymph node; US: ultrasound; ROI: the region of interest; GLCM: gray level co-occurrence matrix; GLRLM: gray level run-length matrix; GLZSM: gray level zone size matrix; ROC: receiver operating characteristic; $A U C$ : area under the receiver operating characteristic (ROC) curves; RF: random forest; SVM: support vector machine; NN: neural network; NB: naïve bayes; Cl: confidence interval

\section{Declarations}

Acknowledgements 
Not applicable.

\section{Authors' contributions}

$\mathrm{MZ}$ and $\mathrm{ZH}$ conceived and designed the study. PW, LQ and HW carried out data

collection. WC, HW and PW conducted data analysis. WC, ZH, PW, DL, JZ and ZD

contributed to interpretation of the data. ZH and PW drafted the manuscript. MZ, WC, ZD, and DL revised the manuscript. All authors read and approved the final version of this manuscript.

\section{Funding}

This study was funded by the National Natural Science Foundation of China (81871337), Medical Science Research Program of Zhejiang Province (2020369811), Hangzhou Social Development Project of Science and Technology Commission (20180533B39), Key Laboratory of Clinical Cancer Pharmacology and Toxicology Research of Zhejiang Province (2020E10021), the Key Project of Scientific and Technological Innovation in Hangzhou (20131813A08). The funder body had no role in the design of the study and collection, analysis, and interpretation of data or in writing the manuscript.

\section{Availability of data and materials}

All data analyzed during this study are available from the corresponding author on reasonable request.

\section{Ethics approval and consent to participate}

This study was approved by the Ethics Committee of the Affiliated Hangzhou First

People's Hospital, Zhejiang University School of Medicine, and Hangzhou Cancer Hospital. The requirement for informed consent was waived given the retrospective nature of the data, in accordance with the national legislation and institutional requirements. All patients data were treated following the local privacy regulations.

\section{Consent for publication}

Not applicable.

\section{Competing interests}

The authors declare that they have no competing interests.

\section{References}

1. Siegel RL, Miller KD, Jemal A. Cancer statistics, 2017. Ca Cancer J Clin. 2017;67(1):7-30. 
2. Noone AM, Cronin KA, Altekruse SF, Howlader N, Lewis DR, Petkov VI, Penberthy L. Cancer incidence and survival trends by subtype using data from the surveillance epidemiology and end results program, 1992-2013. Cancer Epidemiol Biomarkers Prev. 2017;26(4):632-41.

3. Liu Y, Wang Y, Zhao K, Li D, Chen Z, Jiang R, Wang X, He X. Lymph node metastasis in young and middle-aged papillary thyroid carcinoma patients: a SEER-based cohort study. BMC Cancer. 2020;20(1):181.

4. Hughes DT, Haymart MR, Miller BS, Gauger PG, Doherty GM. The most commonly occurring papillary thyroid cancer in the United States is now a microcarcinoma in a patient older than 45 years. Thyroid. 2011;21(3):231-36.

5. Ardakani AA, Rasekhi A, Mohammadi A, Motevalian E, Najafabad BK. Differentiation between metastatic and tumour-free cervical lymph nodes in patients with papillary thyroid carcinoma by grey-scale sonographic texture analysis. Pol J Radiol. 2018;83:e37-46.

6. Siddiqui S, White MG, Antic T, Grogan RH, Angelos P, Kaplan EL, Cipriani NA. Clinical and pathologic predictors of lymph node metastasis and recurrence in papillary thyroid microcarcinoma. Thyroid. 2016;26(6):807-15.

7. Wang W, Gu J, Shang J, Wang K. Correlation analysis on central lymph node metastasis in 276 patients with cNO papillary thyroid carcinoma[J]. Int J Clin Exp Pathol. 2013;6(3):510-5.

8. Spanu A, Nuvoli S, Marongiu A, Gelo I, Mele L, Piras B, Madeddu G. Neck lymph node metastasis detection in patients with differentiated thyroid carcinoma (DTC) in long-term follow-up: a ${ }^{131}$ ISPECT/CT study. BMC Cancer. 2020;20(1):239.

9. Haugen BR, Alexander EK, Bible KC, Doherty GM, Mandel SJ, Nikiforov YE, Pacini F, Randolph GW, Sawka AM, Schlumberger M, Schuff KG, Sherman SI, Sosa JA, Steward DL, Tuttle RM, Wartofsky L. 2015 American thyroid associationmanagement guidelinesfor adult patients with thyroid nodules and differentiated thyroid cancer: the American thyroid association guidelines task force on thyroid nodules and differentiated thyroid cancer. Thyroid. 2016;26(1):1-133.

10. Lesnik D, Cunnane ME, Zurakowski D, Acar GO, Ecevit C, Mace A, Kamani D, Randolph. GW 2014 Papillary thyroid carcinoma nodal surgery directed by a preoperative radiographic map utilizing CT scan and ultrasound in all primary and reoperative patients. Head Neck 36:191-202.

11. Kim E, Park JS, Son KR, Kim JH, Jeon SJ, Na DG. Preoperative diagnosis of cervical metastatic lymph nodes in papillary thyroid carcinoma: comparison of ultrasound, computed tomography, and combined ultrasound with computed tomography. Thyroid. 2008;18(4):411-8.

12. Roh JL, Park JY, Kim JM, Song CJ. Use of preoperative ultrasonography as guidance for neck dissection in patients with papillary thyroid carcinoma. J Surg Oncol. 2010;99(1):28-31.

13. Choi JS, Kim J, Kwak JY, Kim MJ, Chang HS, Kim EK. Preoperative staging of papillary thyroid carcinoma: comparison of ultrasound imaging and CT. Ajr Am J Roentgenol. 2009;193(3):871-8.

14. Lesnik D, Cunnane ME, Zurakowski D, Acar GO, Ecevit C, Mace A, Kamani D, Randolph GW. Papillary thyroid carcinoma nodal surgery directed by a preoperative radiographic map utilizing CT scan and ultrasound in all primary and reoperative patients. Head Neck. 2014;36(2):191-202. 
15. Yeh MW, Bauer AJ, Bernet VA, Ferris RL, Loevner LA, Mandel SJ, Orloff LA, Randolph GW, Steward DL, American Thyroid Association Surgical Affairs Committee Writing Task Force. American thyroid association statement on preoperative imaging for thyroid cancer surgery. Thyroid. 2015;25(1):3-14.

16. Shin JH, Baek JH, Chung J, Ha EJ, Kim JH, Lee YH, Lim HK, Moon WJ, Na DG, Park JS, Choi YJ, Hahn SY, Jeon SJ, Jung SL, Kim DW, Kim EK, Kwak JY, Lee CY, Lee HJ, Lee JH, Lee JH, Lee KH, Park SW, Sung JY. Korean Society of Thyroid Radiology (KSThR) and Korean Society of Radiology. Ultrasonography diagnosis andimaging-based management of thyroid nodules: revised Korean Society of Thyroid Radiology consensus statement and recommendations. Korean J Radiol. 2016;17(3):370-95.

17. Cho SJ, Suh CH, Baek JH, Chung SR, Choi YJ, Lee JH. Diagnostic performance of CT in detection of metastatic cervical lymph nodes in patients with thyroid cancer: a systematic review and metaanalysis. Eur Radiol. 2019;29(9):4635-47.

18. Suh CH, Baek JH, Choi YJ, Lee JH. Performance of CT in the Preoperative Diagnosis of Cervical Lymph Node Metastasis in Patients with Papillary Thyroid Cancer: A Systematic Review and MetaAnalysis. AJNR Am J Neuroradiol. 2017;38(1):154-61.

19. Aerts HJ, Velazquez ER, Leijenaar RT, Parmar C, Grossmann P, Carvalho S, Bussink J, Monshouwer R, Haibe-Kains B, Rietveld D, Hoebers F, Rietbergen MM, Leemans CR, Dekker A, Quackenbush J, Gillies RJ, Lambin P. Decoding tumour phenotype by noninvasive imaging using a quantitative radiomics approach. Nat Commun. 2014;5:4006.

20. Kumar V, Gu Y, Basu S, Berglund A, Eschrich SA, Schabath MB, Forster K, Aerts HJ, Dekker A, Fenstermacher D, Goldgof DB, Hall LO, Lambin P, Balagurunathan Y, Gatenby RA, Gillies RJ. Radiomics: the process and the challenges. Magn Reson Imaging. 2012;30(9):1234-48.

21. Lu W, Zhong L, Dong D, Fang M, Dai Q, Leng S, Zhang L, Sun W, Tian J, Zheng J, Jin Y. Radiomic Analysis for Preoperative Prediction of Cervical Lymph Node Metastasis in Patients with Papillary Thyroid Carcinoma. Eur J Radiol. 2019;118:231-8.

22. Han ZJ, Lei ZK, Li MK, Luo DC, Ding JW. Differential diagnosis value of the ultrasound gray scale ratio for papillary thyroid microcarcinomas and micronodular goiters. Quant Imaging Med Surg. 2018;8(5):507-13.

23. Lei ZK, Li MK, Luo DC, Han ZJ. The clinical significance of ultrasound grayscale ratio in differentiating markedly hypoechoic and anechoic minimal thyroid nodules. J Cancer Res Ther. 2018;14(7):1567-71.

24. Zhang LX, Xiang JJ, Wei PY, Ding JW, Luo DC, Peng ZY, Han ZJ. Diagnostic value of computed tomography(CT) histogram analysis in thyroid benign solitary coarse calcification nodules. J Zhejiang Univ Sci B. 2018;19(3):211-7.

25. Na DK, Choi YJ, Choi SH, Kook SH, Park HJ. Evaluation of cervical lymph node metastasis in thyroid cancer patients using real-time CT-navigated ultrasonography: preliminary study. Ultrasonography. 2015;34(1):39-44.

26. Fisher SB, Perrier ND. The incidental thyroid nodule. CA Cancer J Clin. 2018;68(2):97-105. 
27. Han ZJ, Shu YY, Lai XF, Chen WH. Value of computed tomography in determining the nature of papillary thyroid microcarcinomas: evaluation of the computed tomographic characteristics. Clin Imaging. 2013;37(4):664-8.

28. Liu XW, Ouyang D, Li H, Zhang R, Lv Y, Yang A, Xie CM. Papillary thyroid cancer: dual-energy spectral CT quantitative parameters for preoperative diagnosis of metastasis to the cervical lymph nodes. Radiology. 2014;275(1):167-76.

29. Kursa MB, Rudnicki WR. Feature Selection with the Boruta Package. J Stat Softw. 2010;36:1-13.

30. Oliver Kramer. Machine Learning for Evolution Strategies. Switzerland: Switzerland Springer International Publishing; 2016.

31. Kim SY, Lee E, Nam SJ, Kim EK, Moon HJ, Yoon JH, Han KH, Kwak JY. Ultrasound texture analysis: Association with lymph node metastasis of papillary thyroid microcarcinoma. Plos One. 2017;12(4):e0176103.

32. Liu T, Ge X, Yu J, Guo Y, Wang Y, Wang W, Cui L. Comparison of the application of B-mode and strain elastography ultrasound in the estimation of lymph node metastasis of papillary thyroid carcinoma based on a radiomics approach. Int J Comput Assist Radiol Surg. 2018;13(10):1617-27.

33. Lee JH, Ha EJ, Kim JH. Application of deep learning to the diagnosis of cervical lymph node metastasis from thyroid cancer with CT. Eur Radiol. 2019;29(10):5452-7.

34. Yu H, Buch K, Li B, O'Brien M, Soto J, Jara H, Anderson SW. Utility of texture analysis for quantifying hepatic fibrosis on proton density MRI. J Magn Reson Imaging. 2015;42(5):1259-65.

\section{Figures}




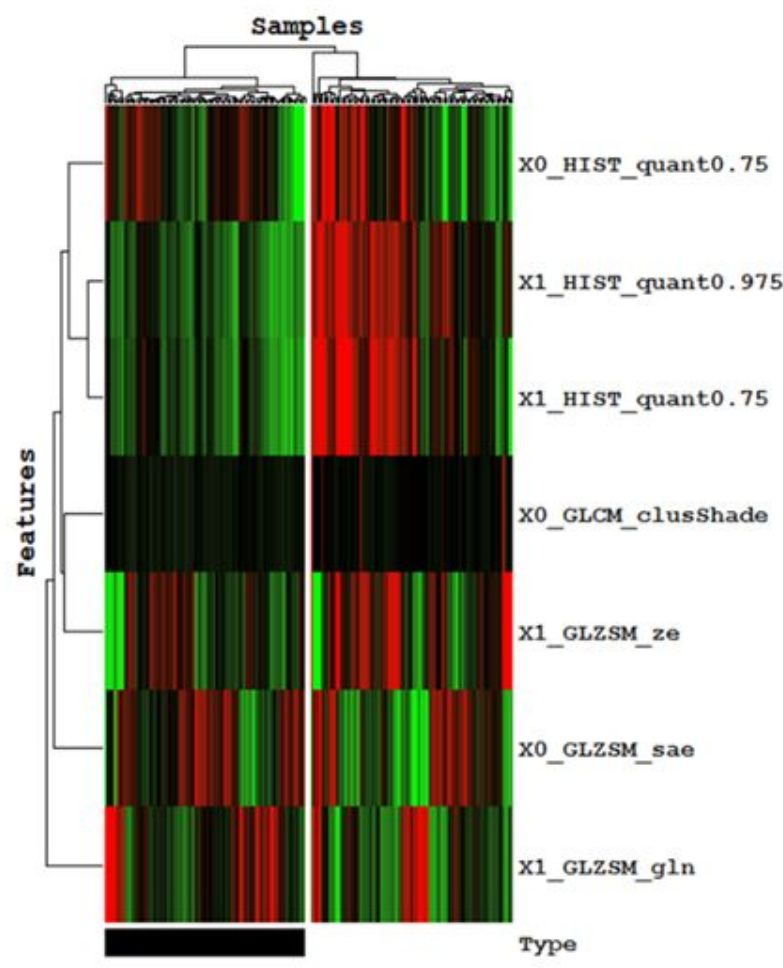

A

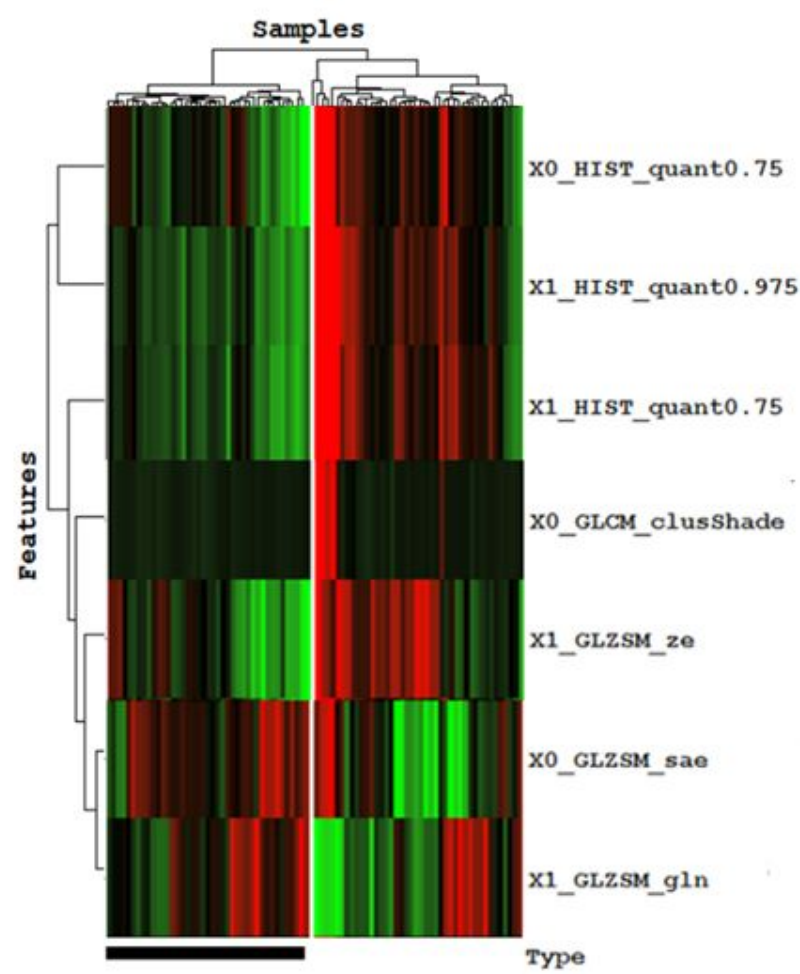

B

\section{Figure 1}

Heat distribution diagram of key features after feature screening: (A) Heatmap for institute 1 data. (B) Heatmap of institute 2 data.

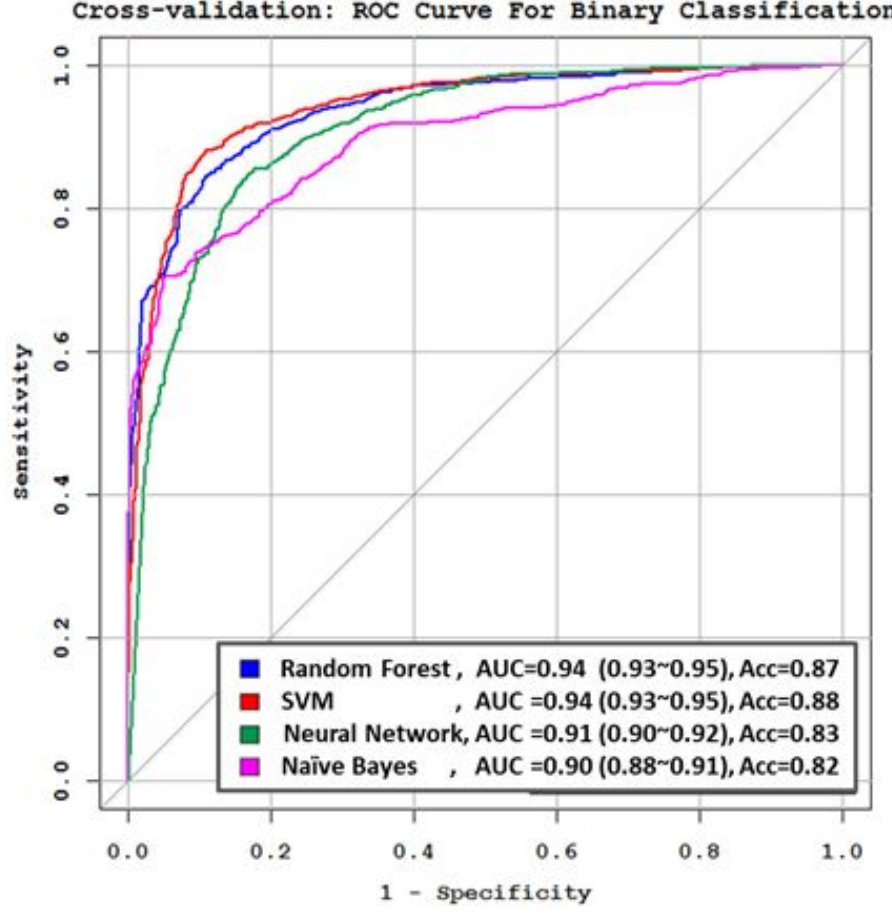

A

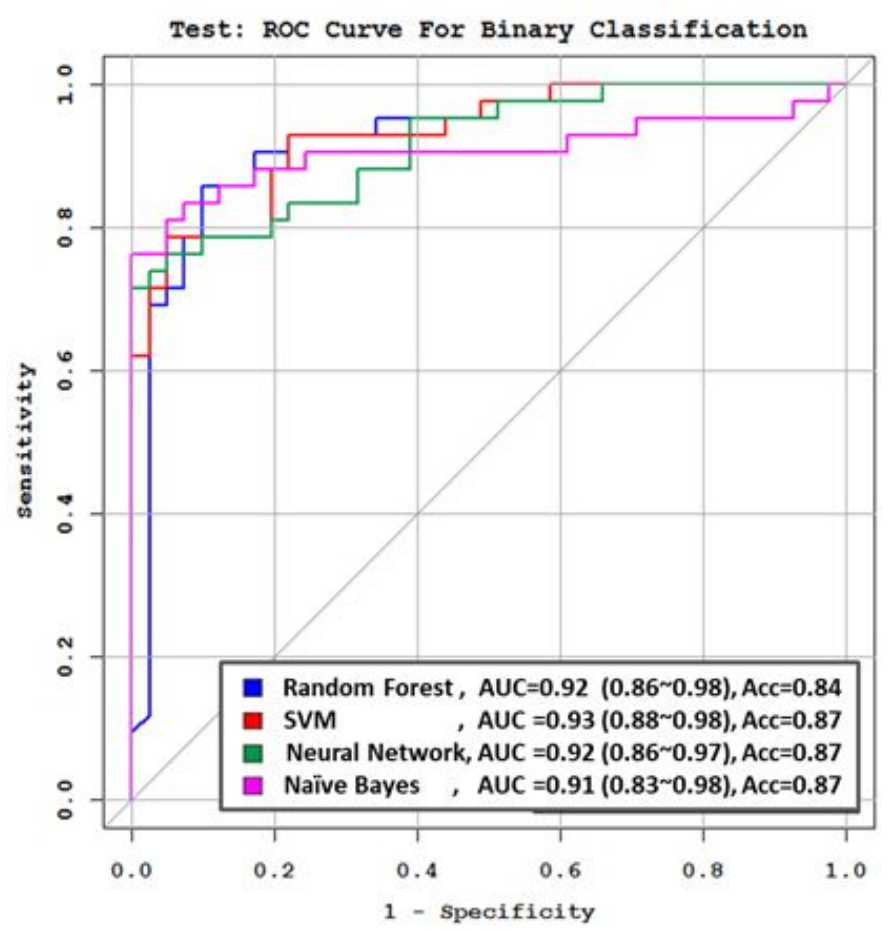

B 
Figure 2

Receiver operating characteristic (ROC) curves of the four the radiomic algorithms for detection of LN metastases: (A) 10-fold cross-validation. (B) External validation. 